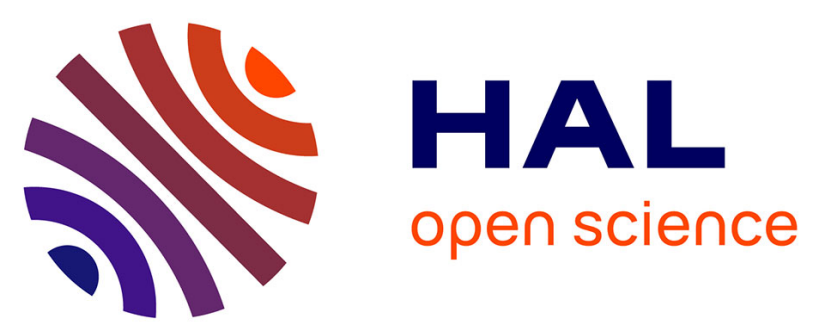

\title{
Conductivity of natural and modified DNA measured by Scanning Tunneling Microscopy. The effect of sequence, charge and stacking
}

Irena Kratochvílová, Karel Král, Martin Bunček, Alena Víšková, Stanislav Nešpůrek, Anna Kochalska, Tatiana Todorciuc, Martin Weiter, Bohdan

Schneider

\section{To cite this version:}

Irena Kratochvílová, Karel Král, Martin Bunček, Alena Višková, Stanislav Nešpůrek, et al.. Conductivity of natural and modified DNA measured by Scanning Tunneling Microscopy. The effect of sequence, charge and stacking. Biophysical Chemistry, 2008, 138 (1-2), pp.3. 10.1016/j.bpc.2008.08.005 . hal-00501716

\section{HAL Id: hal-00501716 https://hal.science/hal-00501716}

Submitted on 12 Jul 2010

HAL is a multi-disciplinary open access archive for the deposit and dissemination of scientific research documents, whether they are published or not. The documents may come from teaching and research institutions in France or abroad, or from public or private research centers.
L'archive ouverte pluridisciplinaire HAL, est destinée au dépôt et à la diffusion de documents scientifiques de niveau recherche, publiés ou non, émanant des établissements d'enseignement et de recherche français ou étrangers, des laboratoires publics ou privés. 


\section{Accepted Manuscript}

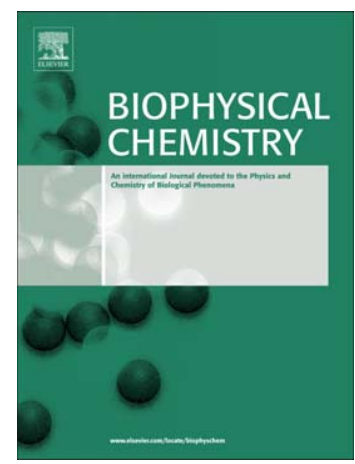

Conductivity of natural and modified DNA measured by Scanning Tunneling Microscopy. The effect of sequence, charge and stacking

Irena Kratochvílová, Karel Král, Martin Bunček, Alena Víšková, Stanislav Nešpůrek, Anna Kochalska, Tatiana Todorciuc, Martin Weiter, Bohdan Schneider

PII: $\quad$ S0301-4622(08)00166-X

DOI: $\quad$ doi: $10.1016 /$ j.bpc.2008.08.005

Reference: $\quad$ BIOCHE 5145

To appear in: Biophysical Chemistry

Received date: 1 June 2008

Revised date: $\quad 12$ August 2008

Accepted date: 16 August 2008

Please cite this article as: Irena Kratochvílová, Karel Král, Martin Bunček, Alena Víšková, Stanislav Nešpůrek, Anna Kochalska, Tatiana Todorciuc, Martin Weiter, Bohdan Schneider, Conductivity of natural and modified DNA measured by Scanning Tunneling Microscopy. The effect of sequence, charge and stacking, Biophysical Chemistry (2008), doi: $10.1016 / j . b p c .2008 .08 .005$

This is a PDF file of an unedited manuscript that has been accepted for publication. As a service to our customers we are providing this early version of the manuscript. The manuscript will undergo copyediting, typesetting, and review of the resulting proof before it is published in its final form. Please note that during the production process errors may be discovered which could affect the content, and all legal disclaimers that apply to the journal pertain. 


\title{
Conductivity of natural and modified DNA measured by Scanning Tunneling Microscopy. The effect of sequence, charge and stacking.
}

Irena Kratochvílová ${ }^{1 *}$, Karel Král ${ }^{1}$, Martin Bunček ${ }^{2}$, Alena Višková ${ }^{2}$, Stanislav Nešpůrek ${ }^{3}$, Anna Kochalska ${ }^{3}$, Tatiana Todorciuc ${ }^{3}$, Martin Weiter ${ }^{4}$, Bohdan Schneider ${ }^{5}$

${ }^{1}$ Institute of Physics, ASCR, v.v.i., Na Slovance 2, CZ-182 21 Prague, Czech Republic; email: krat@fzu.cz

${ }^{2}$ GENERI BIOTECH s.r.o., Machkova 587, CZ-500 11 Hradec Kralové, Czech Republic; email: martin.buncek@generi-biotech.com

${ }^{3}$ Institute of Macromolecular Chemistry ASCR, v.v.i., Heyrovského nám. 2, CZ-162 06 Prague 6, Czech Republic; email: nespurek@imc.cas.cz

${ }^{4}$ Faculty of Chemistry, Brno University of Technology, Purkyňova 118, CZ-612 00 Brno, Czech Republic; email:weiter@fch.vutbr.cz

${ }^{5}$ Institute of Biotechnology AS CR, v.v.i., Vídeňská 1083, CZ-142 20 Prague, Czech Republic; email: bohdan@img.cas.cz

* corresponding author

\begin{abstract}
The conductivity of DNA covalently bonded to a gold surface was studied by means of the STM technique. Various single- and double-stranded 32-nucleotide-long DNA sequences were measured under ambient conditions so as to provide a better understanding of the complex process of chargecarrier transport in natural as well as chemically modified DNA molecules. The investigations focused on the role of several features of DNA structure, namely the role of the negative charge at the backbone phosphate group and the related complex effects of counterions, and of the stacking interactions between the bases in Watson-Crick and other types of base pairs. The measurements have indicated that the best conductor is DNA in its biologically most relevant double-stranded form with Watson-Crick base pairs and charged phosphates equilibrated with counterions and water. All the studied modifications, including DNA with non-Watson-Crick base pairs, the abasic form, and especially the form with phosphate charges eliminated by chemical modifications, lower the conductivity of natural DNA.
\end{abstract}

Key Words: DNA conductivity, Charge transport in molecular systems, STM, Electronic properties of biomolecules 


\section{Introduction}

The DNA molecule, containing the genetic code of all living species, has recently become a center of great attention on the part of chemists and physicists, one of the reasons being DNA's potential use in nanoelectronic devices [1-4], both as a template for assembling nanocircuits and as an active element of such circuits. Charge migration along DNA molecules has attracted scientific interest for more than fifty years. The reports on the possible high rates of charge transfer between the donors and acceptors through DNA, obtained from solution chemistry experiments [5], have triggered a series of direct electrical transport measurements on bundles and networks, because a truly conducting form of DNA would have a major impact on the developments in nanotechnologies.

Extended electronic states of DNA could play an important role in biology, e.g. through the processes of DNA-damage sensing or repairing via long-range charge transfer [6-7]. The prevailing DNA architecture, the double helix, has well stacked, nearly parallel bases with overlapping $\pi$ electron systems. Such $\pi$-electron systems may be good candidates for long-distance and onedimensional (linear) charge transport [8]. Several authors have indicated that DNA conducts electric charge via the hopping mechanism [9-11], but the electronic properties of DNA remain controversial. Charge-transfer reactions and conductivity measurements exhibit a large variety of possible electronic behavior, ranging from Anderson and band-gap insulators to effective molecular wires and induced superconductors. Indeed, understanding the conductance of a complicated polyelectrolyte aperiodic system is in itself a major scientific problem [12-16].

Historically, two basic experimental approaches have been pursued to investigate conduction through molecules: (i) contacting isolated single molecules or molecules in thin films, and (ii) studying transport in thick films and devices, such as organic thin-film transistors or light-emitting diodes [17, 18]. The optimal experimental setup is to position isolated molecules between two electrical contacts, but this is very difficult to implement and especially difficult to verify. Working with the ordered arrays of parallel $\pi$-conjugated molecules, where in principle the binding to the substrate can be precisely controlled, offers a possibility to measure the individual molecules by making use of the scanning-tunneling-microscope (STM) tip. STM experiments have already been proven as a very suitable tool for investigating ordered monolayer films, making it possible to contact one or more molecules and obtain images of the structural characteristics of the film [19, 20]. The experiments have also been used to investigate room-temperature electronic properties of twelve base-pair $\mathrm{d}(\mathrm{GC})_{12}-\mathrm{d}(\mathrm{GC})_{12}$ DNA molecules attached to the gold surface by a thiol link [21]. The STM/STS experiments offer a novel way of probing the electronic properties of biomolecules on surfaces on the atomic level.

In this work, we have used the STM technique in order to study the conductivity of several oligonucleotide sequences in single- and double-stranded forms under ambient conditions. The purpose of these investigations was to understand the complex processes of charge transport through DNA molecules and to dissect the role of the sugar-phosphate backbone, and of its charged phosphate group in particular, as well as of base stacking in the same strand and across strands. To this end, some of the studied oligonucleotide sequences were designed to form non-Watson-Crick base pairs, with the aim of making it possible to estimate the role of these mutation-causing base pairs. The role of the bases and of their stacking interactions in DNA conductivity was also studied at a more fundamental level by removing two or three nitrogenous bases at the central steps of a few sequences forming modified abasic nucleotides. Other sequences contain chemically modified 
phosphate groups, which eliminate their charge (Figure 1). Such modifications make it possible to see the roles of the charged backbone and of the alkali-metal and alkaline-earth-metal counterions on conductivity.

\section{Experimental}

\subsection{Material}

STM experiments were performed on 32-mer oligonucleotides of various sequences, some of which had been chemically modified. For further reference, all the measured oligonucleotides, including their labels, are listed in Table 1. The sequences were designed to investigate the differences: i) between the single-stranded (ss) and double-stranded (ds) DNA of C/G-rich composition, ii) between the single-stranded (ss) and double-stranded (ds) DNA of A/T-rich composition, iii) between dsDNA with only canonical Watson-Crick and containing non-Watson-Crick ("mismatched") base pairs, and iv) between DNA with sequences composed of only natural (non-modified) nucleotides and chemically modified nucleotides. Further, two types of chemical modifications were studied: i) the elimination of nitrogenous bases, thus creating abasic nucleotides, in order to be able to gauge the influence of the stacked bases on the DNA conductivity, and ii) the replacement of the charged phosphate group with its neutral surrogate, which makes it possible to estimate the role of the phosphate charge on the DNA conductivity. The investigated DNA samples (Table 1) were measured in pairs selected in such a way that only one parameter would vary in a pair (see Fig. 1 for the chemical structures of the nucleotides). For instance, the phosphate charge present in the standard oligonucleotide (Fig. 1, label A) is eliminated in DNA E of the identical sequence, the sequences differ between $\mathrm{A}$ and $\mathrm{B}$, or between $\mathrm{C}$ and its abasic mutant $\mathrm{D}$.

The sequences were selected so that they would not form such special structural features as loops or complex tertiary structures. Guanosine nucleotides were evenly distributed as every fourth base in the mixed sequences. The pure G/C (Tab. 1, ss: A and ds: 1) and A/T (Tab. 1, B and 2) oligonucleotides were designed for the evaluation of the effect which the guanine base might have on the overall DNA conductivity. The $\mathrm{C} / \mathrm{G}, \mathrm{T} / \mathrm{A}$ and the mixed $\mathrm{C} / \mathrm{G} / \mathrm{T} / \mathrm{A}$ sequences were prepared as single-stranded and double-stranded with their respective complementary sequences in order to evaluate the effect of single- versus double-stranded DNA forms. So as to ascertain the influence of the heterocyclic nitrogenous bases on the overall charge transport in DNA, we compared the conductivity of the mixed $\mathrm{C} / \mathrm{G} / \mathrm{T} / \mathrm{A}$ sequence (Tab. 1, C - MIX) containing all four bases in equal proportion with guanine evenly distributed on the one hand with a chain with the same sequences except for three removed bases in the middle (Positions 15-17, Tab. 1, D - MIX_S) on the other. These abasic sequences produce a gap with three base pairs missing in the respective ds sample (Tab. 1, ds 3 and 4). Oligo d(T) where two bases in the middle were substituted by either two guanines or by abasic spacer, making two base gaps (Fig. 1b), were also synthesized. To study the impact of charge in the DNA backbone on the charge transport, all the above sequences were also synthesized with a chemically modified phosphate group, which eliminates the phosphate charge. Thus the sequences were prepared as standard phosphodiesters (Fig. 1a), rendering a negative charge on each phosphate, and also as neutral but still polar p-isopropoxy derivatives (Fig. 1c, Tab. 1, E and NGC). How non-canonical (non-W-C) base pairs affect the conductivity was measured on DNA chains modified by introducing sequences with two non-W-C ("mismatched") pairs (Tab. 1, ds 6 and 7). 
All the sequences were analyzed by MALDI-TOF mass spectroscopy in order to prove their identity and evaluate their purity. The sequences with neutralized phosphates charges ( $\mathrm{p}$-isopropoxy modified) showed a small or undetectable number of $\mathrm{Na}^{+} / \mathrm{K}^{+}$adduct peaks, and having compared them to MALDI-TOF measured concentration of $\mathrm{Na}^{+} / \mathrm{K}^{+}$in charged nucleotides, we concluded that the sequences with uncharged phosphates may contain no or only a very small number of $\mathrm{Na}^{+} / \mathrm{K}^{+}$ counterions associated with the oligonucleotide.

Oligonucleotides were synthesized in GENERI BIOTECH, s.r.o., on an ABI394 synthesizer using standard phosphoramidite chemistry. The standard base phosphoramidites, 5'-thiol modifier C6 and dSpacer CE phosphoramidite were purchased from Glen Research, USA, and used according to the manufacturer's recommendations. The p-isopropoxy derivatives of base amidites (deoxy adenosine (n-bz) p-isopropoxy phosphoramidite, deoxy Cytidine (n-bz) p-isopropoxy phosphoramidite, deoxy guanosine (n-ibu) p-isopropoxy phosphoramidite and thymidine p-isopropoxy phosphoramidite) were purchased from ChemGenes Corporation, USA, and also used according to the manufacturer's recommendations. All oligonucleotide sequences were reverse-phase chromatography purified. The 5 '-thiol-modified oligonucleotides were purified according to the manufacturer recommendations, aliquoted immediately after the purification and kept under argon atmosphere before use to prevent oxidative dimerization of the sulfhydryl groups. All the synthesized oligonucleotide sequences were analyzed by HPLC and MALDI-TOF for quality control. According to the MALDI-TOF analysis, the purity of all sequences was $>98 \%$.

All the chemicals were purchased from Sigma Aldrich, USA. The blocking solution of $1 \mathrm{mM} 2-$ mercaptoethanol in MilliQ water (BS), a hybridization buffer of $100 \mathrm{mM}$ Tris. $\mathrm{HCl} / 100 \mathrm{mM} \mathrm{NaCl}$ in MilliQ water (HB) and a washing buffer of $100 \mathrm{mM}$ Tris. $\mathrm{HCl} / 300 \mathrm{mM} \mathrm{NaCl}$ in MilliQ water (WB) were prepared by dissolving the respective amounts of chemicals in pure MilliQ water, free of DNA/RNA and nucleases (Millipore Inc., USA). The 5'-thiol-modified oligonucleotides were dissolved in MilliQ water to $50 \mu \mathrm{M}$ concentration prior to use. $5 \mu \mathrm{l}$ of $50 \mu \mathrm{M}$ solution of each 5'-thiolmodified oligonucleotide were spotted onto the gold support, forming a drop of ca $3 \mathrm{~mm}$ in diameter. The spotted gold support slides were kept closed in a cartridge with humidified atmosphere created by water-filled basins. This cartridge was incubated at $50^{\circ} \mathrm{C}$ for 16 hours, after which the gold support was washed twice with MilliQ water, and $5 \mu \mathrm{l}$ of $1 \mathrm{mM} 2$-mercaptoethanol were spotted onto the same places as the oligonucleotides. The 2-mercaptoethanol (2-ME) was used to enhance the accessibility of the immobilized probes to the complementary target sequences. The thiol group of 2ME rapidly displaced the weaker absorptive contacts between the DNA nucleotides and the substrate, leaving the probes tethered primarily through the thiol end groups. The spots were "self-aligned", because the gold support is hydrophobic and any water solution put onto the oligonucleotidemodified gold support concentrates in the oligonucleotide-containing spots. The slides were incubated in the same humidified cartridge at $40^{\circ} \mathrm{C}$ for 2 hours, after which the slides were washed 5 times with MilliQ water and dried under high-purity nitrogen (9.6). The gold-support slides with single-stranded oligonucleotide spots were kept in a closed box until use. The gold-support slides with the oligonucleotide spots planned to be analyzed as double-stranded were hybridized with the corresponding complementary oligonucleotides. The complementary oligonucleotides were incubated at $1 \mu \mathrm{M}$ concentration in $\mathrm{HB}$ at $80^{\circ} \mathrm{C}$ for 10 minutes. Immediately after the incubation, $5 \mu \mathrm{l}$ of the corresponding complementary oligonucleotides were dropped onto the particular spots and incubated at $40^{\circ} \mathrm{C}$ for 1 hour, after which the spots were washed twice by WB and finally by MilliQ water. Subsequently, the slides were dried under a stream of nitrogen. The whole process was photographed for the easier alignment of the STM tip onto the oligonucleotide spots. All the slides were kept in a closed box in a cool and dry place before use. 
DNA molecules were attached to the gold surface through a sulfur-gold linkage (Fig. 2) [22]. The ssDNA with the 5'-thiol-modifier were used to produce a thiol group at the 5'-terminus of a synthetic oligonucleotide. Recent neutron reflective studies have indicated that ssDNA oligonucleotides form a compact layer on bare gold. Prior to the formation of thiolated-ssDNA, self-assembled $40 \mathrm{~nm}$ thin $\mathrm{Au}$ (99.99\% purity) monolayers were vacuum-evaporated on single crystalline silicon substrate $<100>$ wafers, which were cut into $1 \mathrm{~cm} \times 1 \mathrm{~cm}$ pieces. Subsequently, the substrates were blown using pure nitrogen and exposed to a solution of thiolated oligonucleotide probes in a sodium chloride buffer.

\subsection{Measurements}

The STM measurements were performed with the NTEGRA Prima NT MDT system under ambient conditions. Both topographic and spectroscopic data were obtained using freshly cut Pt/Ir tips. With STM, for a set point of small voltage $\sim 10 \mathrm{mV}$ and relatively large current $\sim 0.5 \mathrm{nA}$ and thus quite a small tip-sample distance, we observed the topography of the samples. The topographic images showed a considerable difference between the bare gold substrates and the samples with DNA molecules. In the case of molecular layers, specific surface patterns were observed. The occurrence of these patterns was attributed to an etching of the bottom gold layers arising from the reaction of the thiol-groups with the gold atoms, forming dissolvable complexes [23-24].

The DNA layers thickness have been investigated using variable angle spectroscopic ellipsometry (VASE, J.A.Woollam \& co.) working in rotating analyzer mode. The measurements were carried out in the spectral range $300-1100 \mathrm{~nm}$ at three angles of incidence 65,70 and $75^{\circ}$. The structural model was constructed to extract the optical functions of the film from the ellipsometry measurements. Structural model consists of: $\mathrm{Si}$ substrate / Au layer / DNA layer. The $\mathrm{Si}$ - substrate was considered as a semi-infinite medium. The optical constants of Si and Au materials were taken from [25]. The thicknesses of both layers and the optical constants of DNA layer were determined using a direct fitting procedure applied to the experimental ellipsometric data. The DNA molecules were ordered in similar geometric structures with the nearest-neighbor spacing being 1-2 $\mathrm{nm}$. The thickness of the DNA layers, determined by ellipsometry was $8.5-9.0 \mathrm{~nm}$. For 32-nucleotide-long DNA molecules, this length corresponds to the average distance between the neighboring bases of about $0.3 \mathrm{~nm}$, which corresponds to the base-base stacking distance intermediate between the B- and A-DNA forms. The DNA double strand is not likely to be completely straight. The mean inclination of a double helix from the fixed vertical line at the length of 32 base pairs is expected to be about $25^{\circ}$ as can be estimated from the generally accepted persistent length $\mathrm{P}$ of a dsDNA of "random sequence", $P \sim 150$ base pairs. A confrontation of the ellipsometry measurement and the persistent length of a DNA double strand leads to a conclusion that DNA molecules are oriented approximately perpendicularly to the Au substrate.

In all cases, we qualitatively compared conductivity of two types of oligonucleotides. In our experimental setting the tip - sample distance was necessary and not easy to control. Firstly, we worked in constant height mode in very small area on both sides of the compared oligonucleotides type border. Secondly, beside current-voltage $(I(V))$ characteristics we also measured $I(h)$ curves $(I-$ current, $h$-distance between the tip and the surface) at the same points. $I(h)$ curves have the advantage of being normalized and in [26] new approach for reading sequence of DNA molecule via tunnel-current decay has been demonstrated. The $I(h)$ decay consists of two regions: the area of very short-range chemical interactions between the end of the tip and the terminate of the molecule plus 
the area of tunneling through the barrier between the tip and sample. The current initially decays slowly and then more rapidly. The $I(h)$ curves are standardly fitted with two exponentials:

$i=i_{0} \exp \left(-\beta_{1} h\right), 0<h<h_{c}$

$i=i\left(h_{c}\right) \exp \left(-\beta_{2}\left(h-h_{c}\right)\right), h_{c}<h$.

Value of $\beta_{2}$ has been found in [26] as corresponding to decay constant of tunneling current. Thus, it can be supposed that at the breakpoint $\left(h_{c}\right)$ the interactions typical for tip extremely close to the substrate are broken and the tunnel current becomes dominant. The same position of the breakpoint and decay constants $\left(\beta_{2}, \beta_{1}\right)$ means the same tip-surface interaction conditions. Working with different set points we can arrange the position of the tip in the proper interaction territory so that the distance between the tip and the DNA terminate is under control. Comparing histograms of the decay constants and $I(h)$ curves breakpoints $\left(h_{c}\right)$ measured on various DNA samples we controlled position of the tip above the compared DNA modifications.

In our experiments, we have measured current passing through the molecules so close to the tip that their contribution is at set voltage higher than the noise. For tip very close to the molecules (defined by the set point value) and very low voltages the number of the molecules contributing to the total current is as small as possible. Current-voltage characteristics were recorded for various feedback voltage and current set points, i.e. for different initial sample-tip distances. In all the experiments, the STM tip acted as the electrical contact on the "top" side of the assembled monolayer of the DNA molecules, whereas the supporting gold substrate acted as the other "bottom" contact. The distance between the top of the molecules and the STM tip was for all the set points $2.5-4 \AA$. The $I(V)$ and $I$ (h) curves were measured at three different set points: $0.1 \mathrm{nA}, 0.1 \mathrm{~V} ; 0.2 \mathrm{nA}, 0.1 \mathrm{~V} ; 0.5 \mathrm{nA}, 0.1 \mathrm{~V}$, corresponding to three different sample-tip separations, using the same strategy for all the samples. The experimental conditions were controlled using $I(h)$ decay analysis and thus we were able to distinguish between the area of tunneling current and current passing through chemically interacted systems. The experimental setup (tip very close to the surface) allows us to expect that the current flows mainly through the molecule nearest to the tip without significant lateral intermolecular contribution to conduction. Two hundred consecutive $I(V)$ sweeps in both voltage directions were taken on each sample (Table 1). Final results presented in this paper are based on the average of a set from the collected $I(V)$ curves. Only those curves which were not affected much by drift of the STM were included in the statistics.

All the results taken into the consideration showed the same trends, i.e. the symmetry for both biases, linearity and super-linearity for lower and higher voltages, respectively. Due to large statistics we can (from our measurements) set the quantitative proportion of different DNA systems conductivities assuming approximately equal number of molecular densities.

\section{Results and Discussion}

The results of the measurements are summarized in Table 2 and Figures 3-7. The conductivity measurements for each considered structural and chemical feature and their possible explanations are discussed below. Noise, in the form of height fluctuations, was higher in the case of the topographic 
images of films than in the case of bare gold. All the organic molecules that have been studied by STM until now show symmetry of the $(I(V))$ characteristics. The symmetry of the $I(V)$ characteristics has been explained on a simple model [27].

The conductivity of all 32-nucleotide-long DNA molecules including the sulfur spacer was comparable in the STM experiments. We measured the conductivity of the DNA molecules using a scanning tunneling microscope under ambient conditions, i.e. in a humid air (40\% humidity) environment and room temperature. $\mathrm{Xu}$ et al. [21], who measured DNA samples using ultrahigh vacuum scanning spectroscopy, concluded that DNA was a wide-band-gap insulator. From this comparison, we can see that the absence or at least substantially lower content of water in the ultrahigh vacuum plays a significant role in the charge pathway. In agreement with what Enders et al. pointed out in their summary [28], we have concluded that water molecules supporting the molecular structure improve the conditions for the electrical current passing through the whole DNA molecule.

\section{$3.1 \mathrm{G} / \mathrm{C}$ chain vs. A/T chain}

The Guanine/Cytosine-rich and Adenine/Thymine-rich oligonucleotides (Tab. 1, ds 1 and 2) were selected for the evaluation of the guanine effect on the overall DNA conductivity.

We found that a double-helical sample with $\mathrm{G} / \mathrm{C}$ bases is a better conductor than the double strand made of A/T bases (Fig. 3, Tab. 2). The guanine base is known to be easily oxidized, generating a charge carrier (hole). Once charges, and especially holes, are created in the uniform DNA chain, the hopping charge transport can apparently occur between discrete guanine sites or delocalized (e.g. polaron) domains [29]. Furthermore, the stacking of the adjacent base pairs is also likely to affect the conductivity via the $\pi$-electron overlap. The G-C pairs with more compact stacking compared to that in the A-T pairs have higher conductivity.

\subsection{Double-stranded vs. single-stranded DNA}

Typical $I(V)$ curves of both ss and ds GC sequences (A and 1 in Table 1) are shown in Figure 4. The conductivity of the double-stranded form is higher than that of the single strand when complementary sequences are compared, which is in agreement with the theoretical model of the same system [30]. Single strands can be considered much less structurally regular than the double helices because of their local and especially long-range deviations from the regular helical arrangement. Reduced stacking interactions then decrease the potential overlap of their $\pi$-electron systems and overall conductivity. Also the first solvation shell, known to play a crucial role in the structural integrity of the double-helical DNA $[31,32]$, is likely to be much less ordered in ss than in dsDNA. Doublestranded DNA has a more regular structure including the first solvation shell, and its long-range periodicity decreases the dispersion of the polarization energy and makes the distribution of hopping states narrower. Considering these effects, the charge-carrier mobility, and thus conductivity, increases in more regular dsDNA systems.

\subsection{Base pairing - Watson-Crick vs. "mismatched" pairs}

Non-Watson-Crick or "mismatched" base pairs are the type of DNA damage where two noncomplementary bases are paired, e.g. adenine with cytosine or thymine with guanine. The role of the non-W-C base pairs and their different stacking in DNA conductivity was estimated by comparing the conductivity of two dsDNA, the canonical 32-nucleotide-long double-stranded DNA molecules (Tab. 1, ds 5 and 8) with analogical systems containing two non-W-C pairs in the middle (Tab. 1, sequences 6 and 7). The unequivocal conclusion is that the molecules containing non-W-C pairs are 
less conductive than the DNA double strands containing only W-C pairs (Fig. 7, Tab. 2). The mismatched molecules are expected to be under mechanical stress, and the disrupted regular periodicity of the stacked W-C pairs and their possibly worse stacking interaction can lead to a decrease in the electron transport efficiency [33].

\subsection{Abasic nucleotides - Structure change and interrupted stacking}

The role of base stacking in DNA conductivity was also estimated by comparing the conductivity of two double-stranded sequences with a mixed G/A/C/T sequence, one containing all bases in W-C pairs (number 3 in Table 1) and the other with the same sequences except for three bases in the middle, at positions 15-17, which were substituted with an abasic nucleotide spacer (number 4 in Table 1). The measurements consistently showed that samples with the abasic spacer had a lower conductivity than the standard DNA sample. The $I(V)$ curves obtained for double-helical DNA samples 3 and 4 are compared in Figure 5. In this case, the conductivity of the sample with the gap in the middle of the sequence was much lower than the conductivity of a standard DNA chain. The same result - a lower conductivity of an abasic dsDNA sample when compared with an intact dsDNA - was obtained in the case of the ds AT chain with two bases missing in the middle (sample 9).

There are no examples of atomic-resolution structures of DNA molecules with two or even three base pairs replaced by an abasic nucleotide. However, structures missing just one base in one of the two strands in a double-helical construct may be significantly deformed, as for example in the crystal structure of human APE1 bound to abasic DNA [34]. Therefore, it is not possible simply to dissect the effect of the overall ds structure and quality of the stacking; both effects, less regular doublehelical structure and sub-optimal stacking interactions, are likely to cause the lower conductivity of the abasic samples. The DNA backbone constitutes a quasi-one-dimensional periodic system, which is essentially independent of the base pair sequence and could allow for extended Bloch states. Nevertheless, conductivity through the backbone seems to be low because of the insulating sugar groups separating the phosphate groups from each other.

\subsection{Phosphate charge}

To study the influence of the DNA negative charge on its conductivity, we synthesized DNA chains with phosphates modified by isopropoxy groups, thus eliminating phosphate negative charges ( $p$ isopropoxy-modified sequences 10,11, 12 in Table 1). The measurements undertaken for the pairs of double-stranded samples 1-10, 2-11, and 3-12 (Tab. 1) demonstrated that in all three cases the pisopropoxy-modified DNA sequences had a lower conductivity than their counterparts of natural DNA (Tab. 2, Fig. 6).

In the polar aqueous environment, DNA forms a double helix with the hydrophobic base planes shielded from the aqueous solvent by the base-pair stacking in its core region with the charged phosphate fully exposed to the solvent. The phosphate negative charges are compensated by partially condensed counterions, typically mono- and bivalent alkali- and alkaline-earth metals such as $\mathrm{Na}^{+}$ and $\mathrm{Mg}^{2+}$. The metals are fully integrated into the solvation shell, which is partially ordered [35-37]. On the basis of a MALDI-TOF analysis, the presence of $\mathrm{Na}^{+}$and $\mathrm{K}^{+}$cations in the p-isopropoxymodified sequences is highly unlikely, and these metal cations probably do not play any role in the conductivity of sequences 10 and 11 . In natural sequences with the presence of $\mathrm{Na}^{+} \mathrm{or}^{\mathrm{Mg}} \mathrm{gg}^{2+}$ counterions, the conductivity is increased partly due to the $\mathrm{Na}^{+}$or $\mathrm{Mg}^{2+}$ states being localized in the large $\pi-\pi^{*}$ energy gap. In the case of sodium, we have small activation gaps (of a few $k T$ ) between the water and sodium states, which could lead to hopping conductivity between the $\mathrm{Na}^{+}$centered 
states. For $\mathrm{Mg}^{2+}$, the occupied water-state energies are not only close to the $\mathrm{Mg}^{2+}$ levels but also very close to the unoccupied $\pi^{*}$ states, leading to the possibility of electron doping of DNA by water or $\mathrm{Mg}^{2+}$. Like in the case of the abasic dsDNA, the lower conductivity of uncharged dsDNA is caused, besides the electronic effects, also by the less regular structure of the uncharged dsDNA.

The shape of the $I(V)$ curves can in general be interpreted as follows: The current passes through the molecules and also tunnels through the tip-molecule area. For low voltages, ohmic behavior was observed due to the Boltzmann distribution of the charge carriers and the constant position of the Fermi level. The higher the voltage is the higher is the current passing through each molecule, resulting in the nonlinear effect of the charge-carrier injection (the shift of the DNA Fermi level to the electronic tail states and their occupation). Therefore, based on our topographic data, we assume that the differences in the conductivity of different samples come from the properties of the individual molecules, not from the specific properties of the molecular monolayers.

\section{Conclusions}

The STM technique was used to study the conductivity of various DNA sequences in single- and double-stranded forms with one strand always covalently bonded to the gold surface. The purpose of our investigations was to provide more insight into the complex process of the charge-carrier transport in DNA molecules - the role of the sugar-phosphate backbone, the role of the counterions complexed around the negatively charged phosphate oxygens, and the effect of the interactions between the bases and their pairs.

The double-helical sample with the G/C bases is a better conductor than the double-stranded sequence containing only $\mathrm{A}$ and $\mathrm{T}$ nucleotides. Easy oxidation of the guanine base makes it possible to generate the charge carriers (holes). The charges, especially holes, created on the uniform DNA chain can move by the hopping charge transport through the discrete guanine sites.

The conductivity of the double-stranded form is greater than that of the single-stranded one when complementary sequences are compared. Single strands can be considered much less structurally regular than the double helices. Regular structures could form better long-range ordering, which decreases the dispersion of the polarization energy and makes the distribution of hopping states narrower. Under these conditions, the charge-carrier mobility, and thus conductivity, in regular systems increases.

We found that the conductivity of the samples with abasic spacers was lower than that of the natural dsDNA samples because of the lack of stacking interactions and likely also because of the structural irregularities and lost periodicity of the double strand.

The DNA chains without charge-bearing groups were less conductive than natural dsDNA. The reasons are likely to be both electronic (the fact that neutral DNA lacks counterions with their effect on lowering the energy gaps between nucleotides) and structural (neutral DNA is likely to be distorted, irregular and hence more insulating than the natural dsDNA) [38].

Theoretical computations show that the orbitals occupied by the $\pi$-electrons contribute strongly to the electronic states, which arises from the electronic coupling between the neighboring bases [39, 40]. Unlike the $\pi$-electrons, which can form extended states, water molecules and counterions create localized states of electrons, resulting in the hopping mechanism of conduction, with the inclusion of the electron-phonon interactions. The DNA molecule contains electronic states, which have an extended character and in which the electrons easily carry a ballistic current, and localized states of 
electrons, mediating the hopping mechanism of conduction. These two rather complementary pictures of conduction, $\pi$-electron overlap and hopping through energetically near states, may fit together.

Based on our measurements, we can conclude that the best conductor is the natural double-stranded DNA form with its bases forming Watson-Crick pairs and surrounded by counterions and water molecules. Since electronic states are strongly connected with molecular structure and DNA structure changes with base sequences, type and concentration of counterions, and relative humidity, the electronic states are expected to depend on all of the following parameters:

1. change of the regular structure;

2. water (as a possible ion conductor) amount;

3. counterion presence; in the case of DNA containing sodium, $\mathrm{Na}^{+}$can mediate hopping conductivity between the $\mathrm{Na}^{+}$-centered states; for DNA containing magnesium, there is a possibility of electron doping of DNA by water or $\mathrm{Mg}^{2+}$ states.

Acknowledgements. This work was supported by the Grant Agency of the Academy of Sciences of the Czech Republic (Grants No. KAN 200100 801, KAN 401770 651, KAN 400720701 and COST OC 137), the Czech Science Foundation (Grant No. 203/08/1594) and by the European Commission through the Human Potential Programme (Marie-Curie RTN BIMORE, Grant No. MRTN-CT-2006-035859). 
a)<smiles>[R]OC[C@H]1O[C@@H](n2ccc(N)nc2=O)C[C@@H]1OP(=O)([O-])OC[C@H]1O[C@@H](n2cc(C)c(=O)[nH]c2=O)C[C@@H]1OP(=O)([O-])OC[C@H]1O[C@@H](n2cnc3c(N)ncnc32)C[C@@H]1OP(=O)([O-])OC[C@H]1O[C@@H](n2cnc3c(=O)[nH]c(N)nc32)C[C@@H]1O[R]</smiles>

b)<smiles>[R]OC[C@H]1O[C@@H](n2ccc(N)nc2=O)C[C@H]1OP(=O)([O-])OC[C@H]1O[C@@H](n2cc(C)c(=O)[nH]c2=O)[C@H](OP(=O)([O-])OC[C@H]2OCC[C@@H]2OP(=O)([O-])OC[C@H]2O[C@@H](n3cnc4c(=O)[nH]c(N)nc43)C[C@@H]2O[R])[C@@H]1CCC</smiles>

c)<smiles>[R]OC[C@H]1O[C@@H](n2ccc(N)nc2=O)C[C@@H]1OP(=O)(OC[C@H]1O[C@@H](n2cc(C)c(=O)[nH]c2=O)C[C@@H]1OP(=O)(OC[C@H]1O[C@@H](n2cnc3c(N)ncnc32)C[C@@H]1OP(=O)(OC[C@H]1O[C@@H](n2cnc3c(=O)[nH]c(N)nc32)C[C@@H]1O[R])OC(C)C)OC(C)C)OC(C)C</smiles>

Fig. 1: The chemical scheme of the studied nucleotides: (a) a natural nucleotide; b) a part of an abasic oligonucleotide with base removed; c) oligonucleotide with its phosphate neutralized by a Pisopropoxy group. 


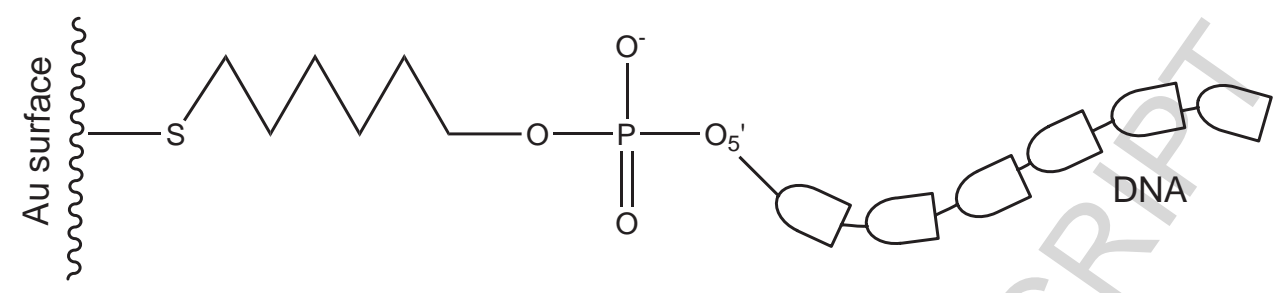

Fig. 2: The scheme of the attachment of the DNA 32-mers to the gold surface.

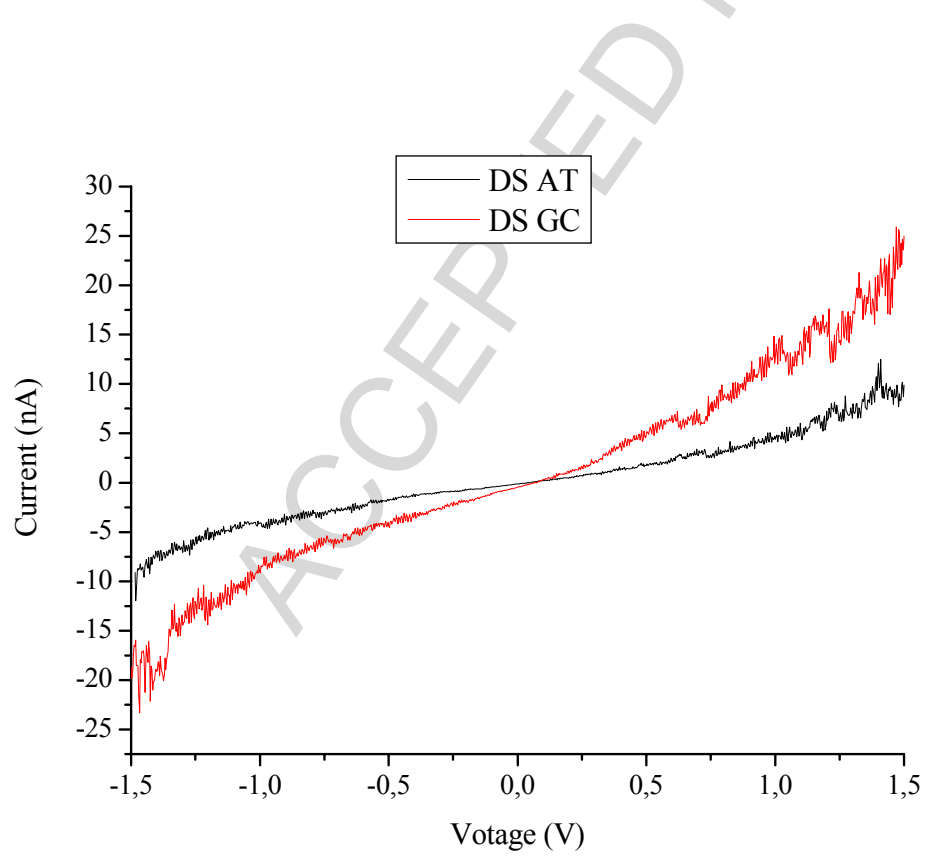

Fig. 3: The typical $I(V)$ curves of DNA GC 32-base double strand (DS GC, see Tab. 1, ds 1) and DNA AT 32-base double strand (DS AT, see Tab. 1, ds 2). Set point $0.1 \mathrm{nA}, 0.1 \mathrm{~V}$, measured in one voltage direction. 


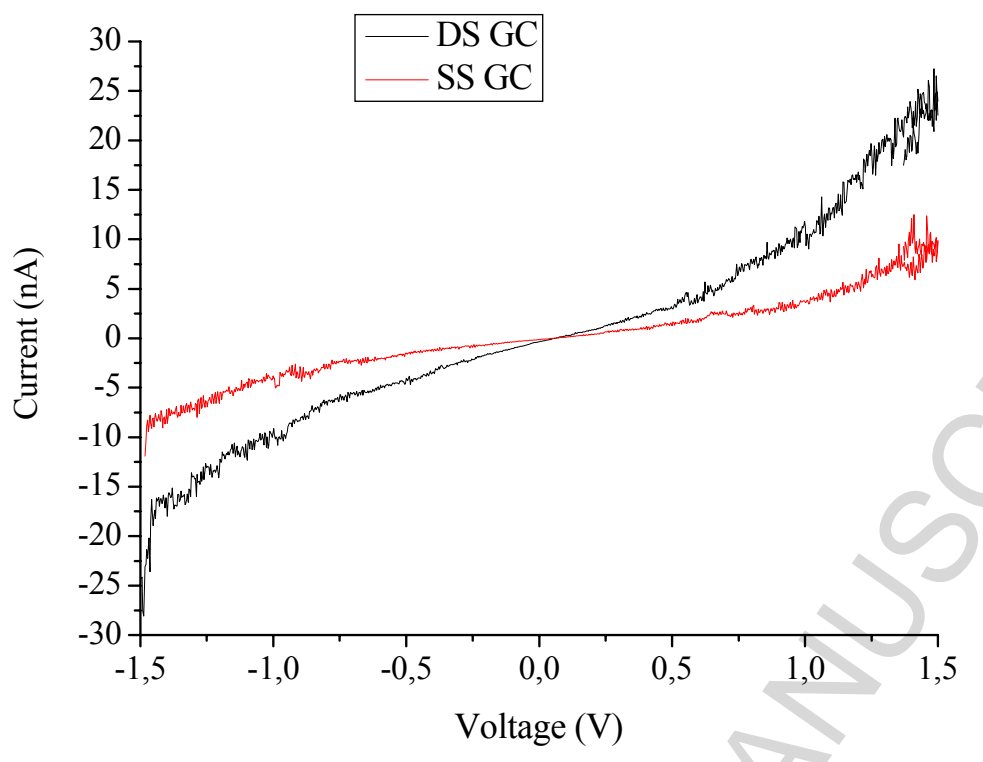

Fig. 4: The typical $I(V)$ curves of DNA GC 32-base single strand (SS GC, see Tab. 1, SS A) and DNA GC 32-base double strand (DS GC, see Tab. 1, ds 1). Set point $0.1 \mathrm{nA}, 0.1 \mathrm{~V}$, measured in one voltage direction.

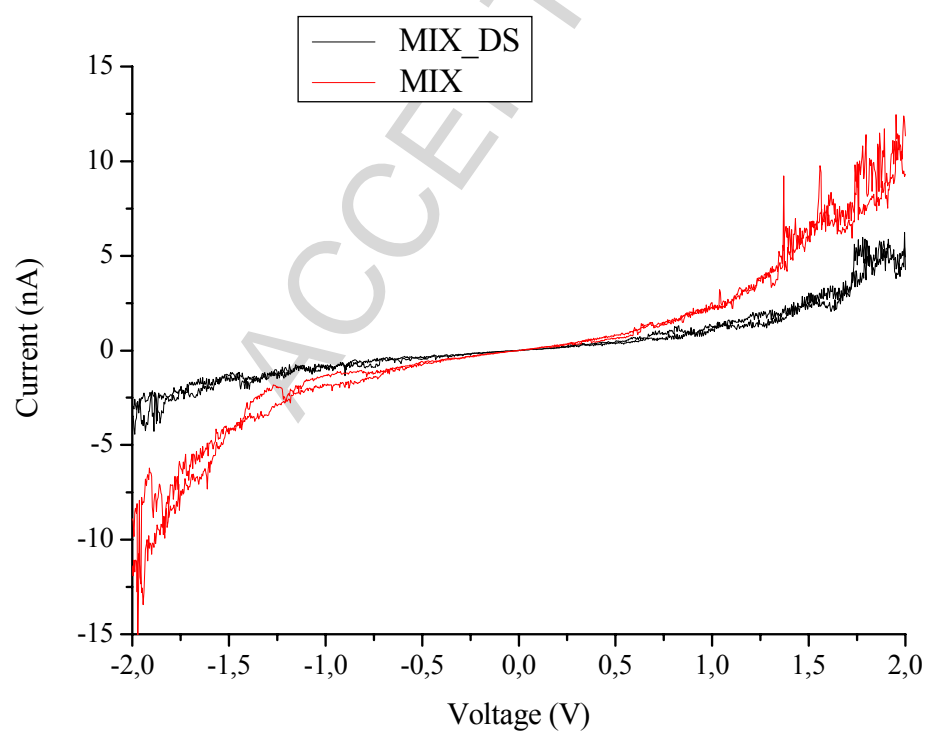

Fig. 5: $I(V)$ curves of double-helical DNA 32-mers with mixed G, A, C, T sequences ( Tab. 1, ds sample 3) and double-helical DNA 32-mers with mixed G, A, C, T sequences with three bases missing in the middle (MIX_DS, see Tab. 1, ds 4). Set point $0.1 \mathrm{nA}, 0.1 \mathrm{~V}$, measured in both voltage directions. 


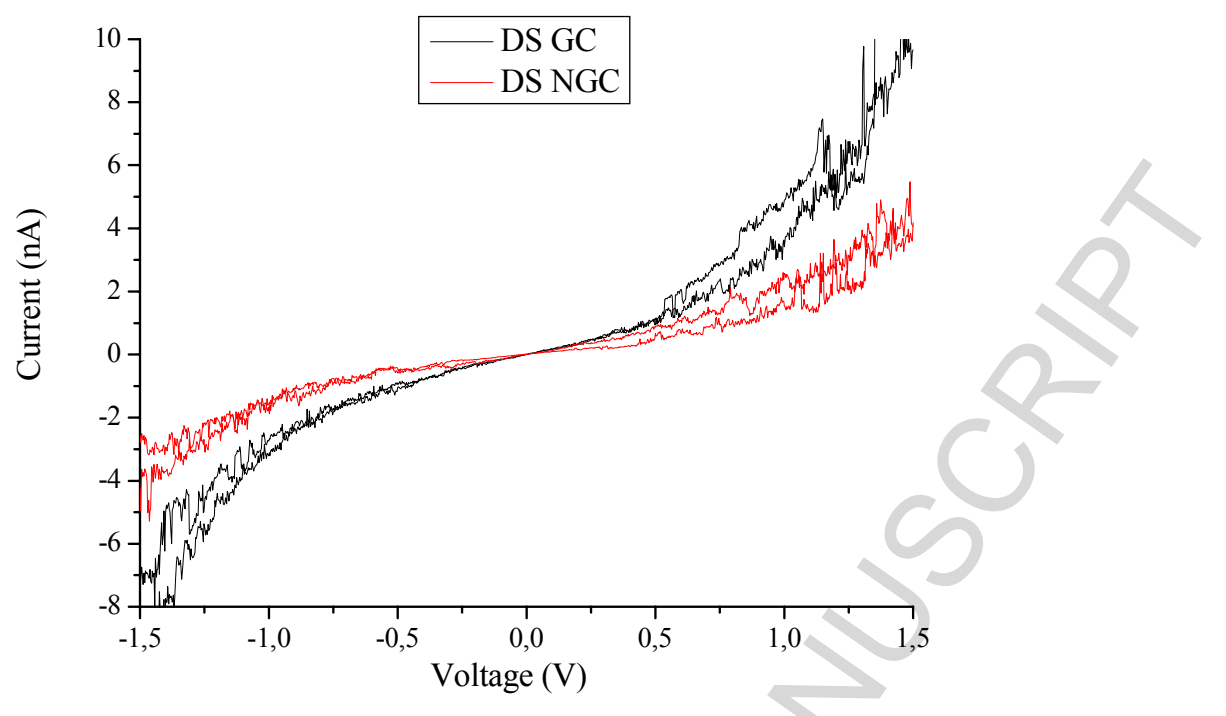

Fig. 6: $I(V)$ curves of double-helical DNA 32-mers with G-C bases without charge-bearing groups on the phosphates - DS NGC (see Tab. 1, ds 10) and standard double-helical DNA 32-mers with G-C bases - DS GC (see Tab. 1, ds 1). Set point $0.1 \mathrm{nA}, 0.1 \mathrm{~V}$, measured in both voltage directions.

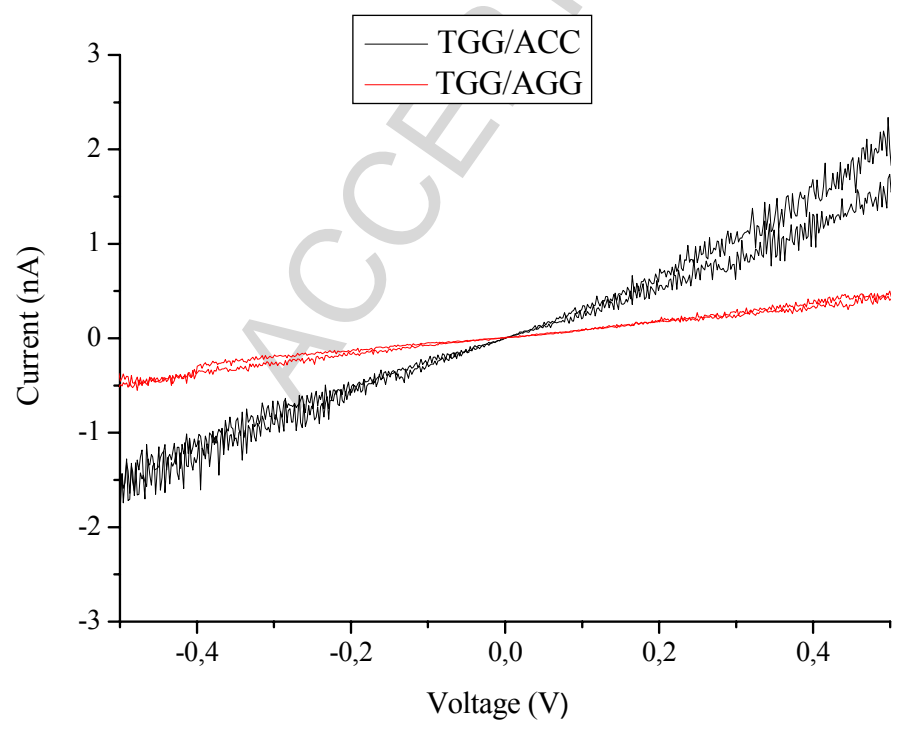




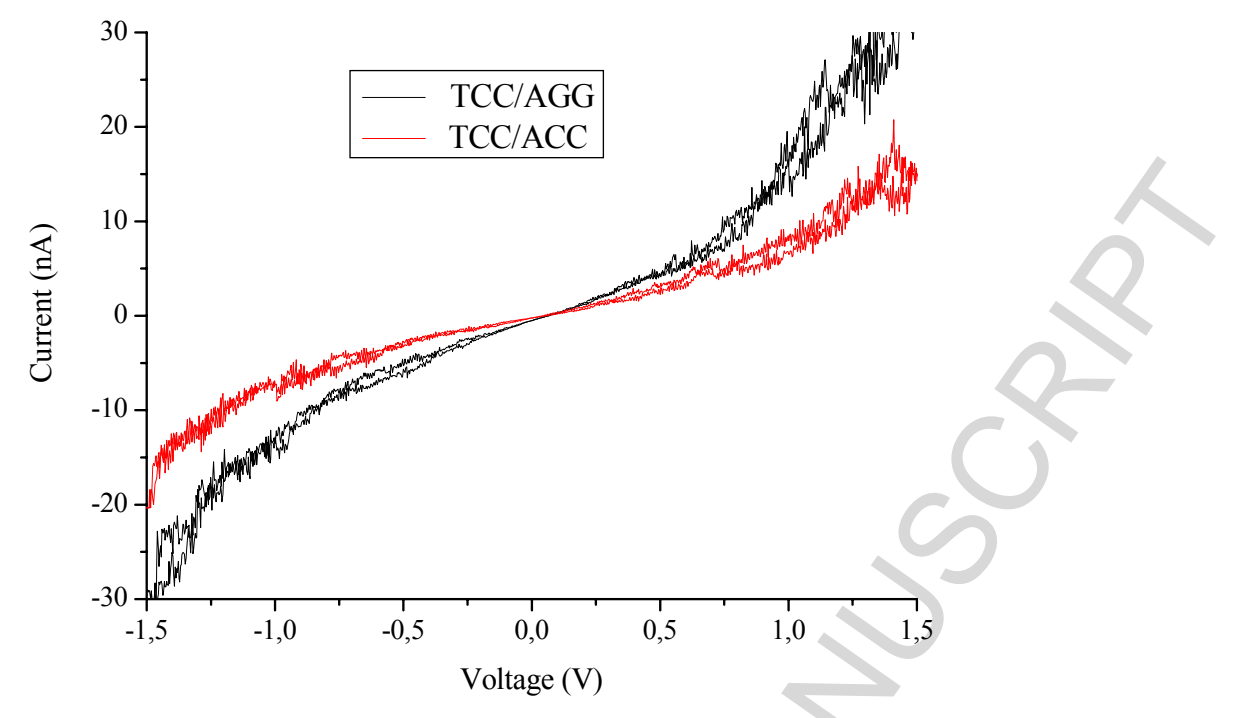

Fig. 7: Typical $I(V)$ curves of DNA molecules containing 4 non-Watson-Crickbase pairs in the middle (TCC vs. ACC, TGG vs. AGG, see Tab. 1, ds 7) and standard dsDNA (TCC vs. AGG, see Tab. 1, ds 8). Set point $0.1 \mathrm{nA}, 0.1 \mathrm{~V}$, measured in both voltage directions. 
Table 1. The investigated DNA sequences. Single-stranded samples are labeled with letters A-E and named, double-stranded DNA are numbered 1-12. G, A, C, T are the symbols that designate the deoxynucleotides with either unmodified backbone (NO "Backbone modification") or the phosphate charge neutralized by an isopropoxy group (Figure 1), whereas the symbols " $x$ " indicate abasic nucleotides. Strands chemically attached to the gold plate start with Au-S. For double-stranded samples, "|" labels the Watson-Crick pair while "₹" indicates a non-Watson-Crick pair.

\begin{tabular}{|l|l|l|c|}
\hline Code & Name & Sequence & Backbone modification \\
\hline A & G/C & Au-S-CGCCGCCGCCGCCGCCGCCGCCGCCGCCGCCG-3' & NO \\
\hline B & A/T & Au-S-ATAATAATAATAATAATAATAATAATAATAAT-3' & NO \\
\hline C & MIX & Au-S-GTTAGCACGATAGTCCGATAGTCAGTCAGTCC-3' \\
\hline D & MIX_dS & Au-S-GTTAGCACGATAGTxXXATAGTCAGTCAGTCC-3' & NO \\
\hline E & NGC & Au-S-CGCCGCCGCCGCCGCCGCCGCCGCCGCCGCCG-3' & uncharged \\
\hline
\end{tabular}

\begin{tabular}{|c|c|c|}
\hline Code & Sequence & $\begin{array}{c}\text { Backbone } \\
\text { modification }\end{array}$ \\
\hline 1 & $\begin{array}{c}\mathrm{Au}-\mathrm{S}-\mathrm{CGCCGCCGCCGCCGCCGCCGCCGCCGCCGCCG-3} \text { ' } \\
|\||\||\||\|\mid\|\|\|\|\|\|\|\|\|\|\|\|\| \\
3^{\prime}-\text { GCGGCGGCGGCGGCGGCGGCGGCGGCGGCGGC-5 ' }\end{array}$ & NO \\
\hline 2 & $\begin{array}{l}\text { Au-S-ATAATAATAATAATAATAATAATAATAATAAT-3 ' } \\
|||||||||||||||||||||||||||||||| \\
\text { 3'-TATTATTATTATTATTATTATTATTATTATTA-5 ' }\end{array}$ & NO \\
\hline 3 & $\begin{array}{c}\text { Au-S-GTtAGCACGAtAgtCCGAtAgtCAGTCAGTCC-3' } \\
|||||||||||||||||||||||||||||| \\
3^{\prime} \text {-CAATCGTGCTATCAGGCTATCAGTCAGTCAGG-5 ' }\end{array}$ & NO \\
\hline 4 & $\begin{array}{c}\text { Au-S-GTtAGCACGAtAgTxxxAtAgtCAGTCAGTCC-3' } \\
|||||||||||||||||||||||||| \\
3^{\prime} \text {-CAATCGTGCTATCAxxxTATCAGTCAGTCAGG-5' }\end{array}$ & NO \\
\hline 5 & 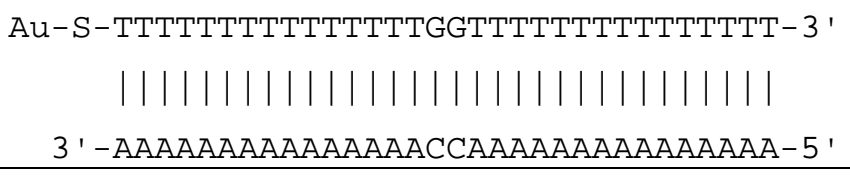 & NO \\
\hline 6 & 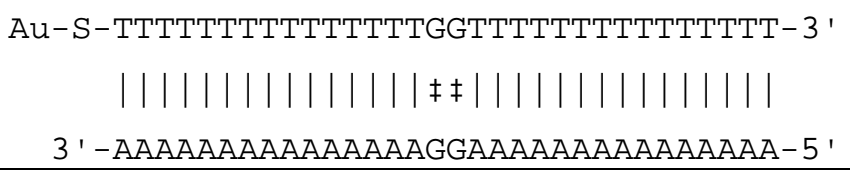 & NO \\
\hline
\end{tabular}




\begin{tabular}{|c|c|c|}
\hline 7 & 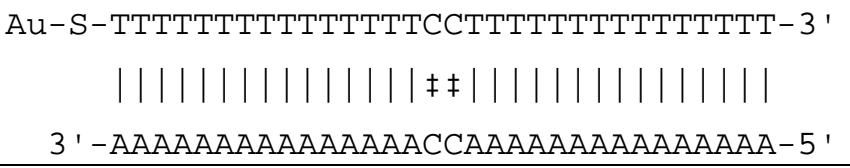 & NO \\
\hline 8 & 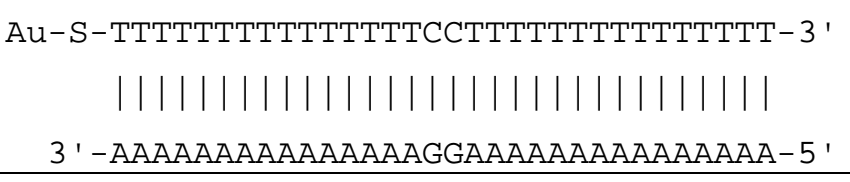 & NO \\
\hline 9 & 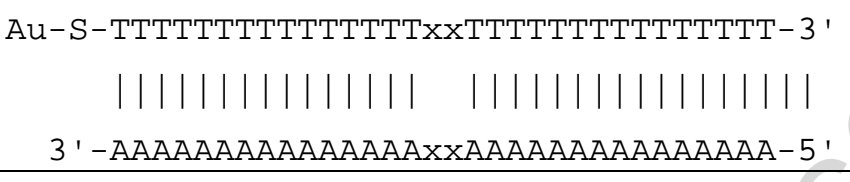 & NO \\
\hline 10 & 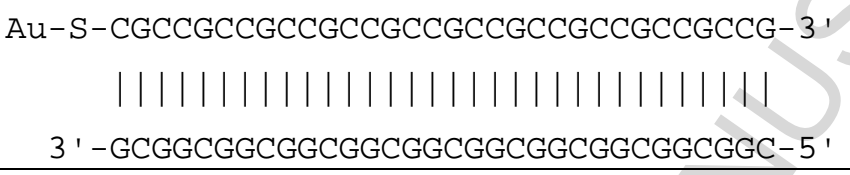 & $\begin{array}{c}\text { Uncharged } \\
\text { P-isopropoxy }\end{array}$ \\
\hline 11 & $\begin{array}{l}\text { Au-S-ATAATAATAATAATAATAATAATAATAATAAT-3' } \\
|||||||||||||||||||||||||||||||| \mid \\
\text { 3'-TATTATTATTATTATTATTATTATTATTATTA-5' }\end{array}$ & $\begin{array}{c}\text { uncharged } \\
\text { P-isopropoxy }\end{array}$ \\
\hline 12 & $\begin{array}{c}\text { Au-S-GTtAGCACGATAGTCCGATAgTCAGTCAGTCC-3' } \\
|||||||||||||||||||||||||||||| \\
3^{\prime}-\text { CAATCGTGCTATCAGGCTATCAGTCAGTCAGG-5' }\end{array}$ & $\begin{array}{c}\text { uncharged } \\
\text { P-isopropoxy }\end{array}$ \\
\hline
\end{tabular}

Table 2: A comparison of the conductivity of DNA samples measured on the same gold plate in one experiment. The codes of DNA samples are listed in Table 1.

\begin{tabular}{|c|c|c|c|c|c|c|c|c|c|c|}
\hline $\begin{array}{c}\text { DNA samples measured in one } \\
\text { experiment }\end{array}$ & $1 / 2$ & $\mathrm{~A} / 1$ & $3 / 4$ & $5 / 9$ & $8 / 9$ & $1 / 10$ & $2 / 11$ & $8 / 7$ & $5 / 6$ & $3 / 12$ \\
\hline $\begin{array}{c}\text { DNA samples with higher } \\
\text { conductivity }\end{array}$ & 1 & 1 & 3 & 5 & 8 & 1 & 2 & 8 & 5 & 3 \\
\hline
\end{tabular}




\section{References:}

[1] S. Záliš, I. Kratochvílová, A. Zambova, J. Mbindyo, T. E. Mallouk and T. S. Mayer, Combined experimental and theoretical DFT study of molecular nanowires negative differential resistance and interaction with gold clusters, Eur. Phys. J. E 18 (2005) 201-206.

[2] I. Kratochvílová, A. Zambova, J. Mbindyo, B. Razavi and J. Holakovský, Current-voltage characterization of alkanethiol self-assembled monolayers in metal nanowires, Mod. Phys. Lett. B 16, (2002) 161-169.

[3] I. Kratochvílová, S. Nešpůrek, J. Šebera, S. Záliš, M. Pavelka, G. Wang, J. Sworakowski, New organic FET-like photoactive device, experiments and DFT modeling, Eur. Phys. J. E., 2008, 25, 299307.

[4] I. Kratochvílová, K. Král, M. Bunček, S. Nešpůrek, T. Todorciuc, M. Weiter, J. Navrátil, B. Schneider, J. Pavluch: Scanning tunneling spectroscopy study of DNA conductivity, Central European Journal of Physics, 1895-1082 (Print) 1644-3608 (Online).

[5] B. Giese : Electron Transfer in DNA, Current Opinion in Chemical Biology 6 (2002) 612-618.

[6] A. Heller Spiers memorial lecture. On the hypothesis of cathodic protection of genes, Faraday Discuss, 116 (2000) 1-13.

[7] B. Giese B and M. Spichty, Long-distance charge transport through DNA: Quantification and extension of the hopping model, Chem.Phys.Chem. 1(2000) 195-198

[8] D.T. Odom, E.A. Dill and J.K. Barton, Robust charge transport in DNA double crossover assemblies, Chem.Biol. 7 (2000) 475-481.

[9] M. E. Nuñez, D.B. Hall and J.K. Barton, Long-range oxidative damage to DNA: effects of distance and sequence, Chem Biol 6 (1999) 85-97.

[10] G.B. Schuster, Long-range charge transfer in DNA: transient structural distortions control the distance dependence, Acc. Chem. Res. 33 (2000) 253-260.

[11] B. Giese, Long-distance charge transport in DNA: the hopping mechanism, Acc Chem Res 33 (2000) 631-636.

[12] R.G. Enders, D.L. Cox, and R.R.P. Singh, Colloquium: The quest for high-conductance DNA, Rev. Modern Phys. 76 (2004) 195-217.

[13] E.B. Starikov, S. Tanaka, N. Kurita, Y. Sengok, T. Natsume, and W. Wenzel, Investigation of a Kubo-formula-based approach to estimate DNA conductance in an atomistic model, Eur.Phys.J.E 18 (2005) 437-445.

[14] M. Taniguchi and T. Kawai, DNA Electronics. Physica E 33 (2006)1-12. 
[15] H. Cohen , C. Nogues, R. Naaman, and D. Porath, Direct measurement of electrical transport through single DNA moleculas of complex sequence, PNAS 102 (2005) 11589-11593.

[16] D. Ullien, H. Cohen and D. Porath, The effect of the number of parallel DNA molecules on electric charge transport through "standing DNA", Nanotechnology 18 (2007) 424015-424018.

[17] H. Cohen, C. Nogues, D. Ullien, S. Daube, R. Naaman and D. Porath, Faraday Discussions 131 (2006) 367-376.

[18] D. Porah, G. Cuniberti and R. Felice, Charge transport in DNA - based devices, Top Curr. Chem. 237 (2004) 183-227.

[19] A.I. Onipko, K. F. Berggren, Y. O. Klymenko, L. I. Malysheva, J. J. W. M. Rosnik, L. J. Geerlings, E. van der Drift, and S. Radelaar, Scanning tunneling spectroscopy on $\pi$-conjugated phenyl-based oligomers: a simple physical model, Phys. Rev. B 61 (2000) 11118-11124.

[20] J.J.W.M. Rosnik, M.A. Blauw, L.J. Geerlings, E. van der Drift, and S. Radelaar, Tunneling spectroscopy and modeling of electron transport in small conjugated azomethine molecules, Phys. Rev. B 62 (2000) 10459-10466.

[21] M.S. Xu, S. Tsukamoto, S. Ishida, M. Kimatura, Y. Arakawa, R.G. Endres and M. Shimoda, Conductance of single thiolated poly(GC)-poly(GC) DNA molecules, Appl. Phys. Lett. 87 (2005) 083902-1-3.

[22] J.J.W.M. Rosink, A. Blauw, L.J. Geerligs, E. van der Drift, B.A.C. Rousseeuw, and S. Radelaar, Growth and characterisation of organic multilayers on gold grown by organic molecular beam deposition, Opt.Mater. 9 (1998) 416-422.

[23] C. Schoenenberger, J.A.M. Sondag-Huethorst, J. Jorritsma, and L.G.J. Fokkink, What Are the Holes in Self-Assembled Monolayers of Alkanethiols On Gold, Langmuir 10 (1994) 611-614.

[24] J.J.W.M. Rosink, A. Blauw, L.J. Geerligs, E. van der Drift, B.A.C. Rousseeuw, and S. Radelaar, Growth and characterisation of organic multilayers on gold grown by organic molecular beam deposition, Opt.Mater. 9 (1998) 416-422.

[25] [ Handbook of Optical Constants of Solids, edited by E. D. Palik, 1985].

[26] Jin He, Lisha Lin, Peiming Zhang and Stuart Lidsay : Identification of DNA Basepairing via Tunnel-Current Decay, Nano Letters, 7 (2007) 3854-3858)

[27] S. Datta, W. Tian, S. Hong, R. Reifenberger, I. Henderson, and C. Kubiak, Current-voltage characteristics of self-assembled monolayers by scanning tunneling microscopy. Phys.Rev.Lett. 79 (1997) 2530-2533.

[28] R.G. Enders, D.L. Cox, and R.R.P. Singh, Colloquium: The quest for high-conductance DNA, Rev. Modern Phys. 76 (2004) 195-217. 
[29] L. Cai, H. Tabata, and T. Kawai, Self-assembled DNA networks and their electrical conductivity, Appl. Phys. Let. 77 (2000) 3105-3106.

[30] E.B. Starikov, S. Tanaka, N. Kurita, Y. Sengok, T. Natsume, and W. Wenzel, Investigation of a Kubo-formula-based approach to estimate DNA conductance in an atomistic model, Eur.Phys.J.E 18 (2005) 437-445.

[31] E. Westhof, Structural bridges in nucleic acids. in Westhof E. (1993). Water and biological macromolecules. Boca Raton, CRC Press.

[32] H.M. Berman and B. Schneider. 1998. Nucleic acid hydration. In Handbook of nucleic acid structure. S. Neidle, ed. Oxford University (1999).

[33] B. Giese, Electron transfer in DNA, Current Opinion in Chemical Biology, 6 (2002) 612-618.

[34] C.D. Mol, T. Izumi, S. Mitra and J.A. Tainer, DNA-bound structures and mutants reveal abasic DNA binding by APE1 and DNA repair coordination, Nature 403 (2000) 451-456.

[35] B. Schneider, and M. Kabelac: Stereochemistry of binding of metal cations and water to a phosphate group, J. Am. Chem. Soc. 120 (1998) 161-165.

[36] B. Halle and V. P. Denisov, Water and monovalent ions in the minor groove of B-DNA oligonucleotides as seen by NMR, Biopolymers, 48 (1998) 210-233.

[37] B. Schneider, K. Patel and H. M. Berman: Hydration of the Phosphate Group in Double-Helical DNA, Biophys. J. 75 (1998) 2422-2434.

[38] R.G. Enders, D. L. Cox, and R.R.P. Singh, Colloquium: The quest for high-conductance DNA, Rev. Modern Phys. 76 (2004) 195-217.

[39] F. Zahid, M. Paulsson and S. Datta, Chapter published in "Advanced Semiconductors and Organic nano-Techniques", edited by H. Morkoc, Academic Press 2003. See also arXiv: CondMat/0208183.

[40] B. Rezek, D. Shin, H. Uetsuka, and Ch. E. Nebel, Microscopic diagnostics of DNA molecules on mono-crystalline diamond, phys. stat. sol. (a), (2007) 1-10 / DOI 0.1002/pssa.200776317. 